\title{
Texture Analysis by the Schulz Reflection Method: Defocalization Corrections for Thin Films
}

\author{
By D. Chateigner, P. Germi and M. Pernet \\ Laboratoire de Cristallographie, Centre National de la Recherche Scientifique, associé à l'Université Joseph \\ Fourier, 166 X, 38042 Grenoble CEDEX 09, France
}

(Received 4 February 1992; accepted 9 June 1992)

\begin{abstract}
A new method is described for correcting experimental data obtained from the texture analysis of thin films. The analysis employed for correcting the data usually requires the experimental curves of defocalization for a randomly oriented specimen. In view of difficulties in finding non-oriented films, a theoretical method for these corrections is proposed which uses the defocalization evolution for a bulk sample, the film thickness and the penetration depth of the incident beam in the material. This correction method is applied to a film of $\mathrm{YBa}_{2} \mathrm{Cu}_{3} \mathrm{O}_{7-\delta}$ on an $\mathrm{SrTiO}_{3}$ singlecrystal substrate.
\end{abstract}

\section{Introduction}

The particular effects of anisotropy in polycrystalline samples are only physically distinguishable if most of their crystallites tend to be aligned along one or more of their crystallographic directions. Such an alignment is called the texture or preferred orientation and is of great interest for the exploitation of magnetic powders, for instance, or, more recently, of high- $T_{c}$ superconductors in the case of bulk, fibre or thin-film samples. For characterizing textures, the mapping of the spatial distribution of crystallographic directions on 'pole figures' is known to be one of the best methods and has been described in two different forms known as the reflection and transmission techniques (Schulz, 1949). For studying thin films on substrates, only the reflection method can be used. In this case, special experimental defocalization corrections are used which need a sample without preferred orientation, where crystallites are randomly distributed (Bunge, 1982; Hermida, 1982; Humbert, 1986; Ortiz \& Hermida, 1981). However, it is very difficult to obtain randomly oriented thin films, especially with highly anisotropic materials and, to our knowledge, the reported studies of films used no special procedures for corrections.

This work presents a theoretical method for these corrections and describes its application to a
$\mathrm{YBa}_{2} \mathrm{Cu}_{3} \mathrm{O}_{7-\delta}$ film on an $\mathrm{SrTiO}_{3}$ single-crystal substrate.

\section{Theoretical study}

The schematic arrangement of the generally used Schulz reflection method is shown in Fig. 1. The texture goniometer allows variation of two Euler angles, $\varphi$ and $\beta$ scans, and the Bragg angle $\theta$. The latter is fixed for the analysis of the spatial distribution of one type of diffracting plane $(h, k, l)$.

The irradiated surface is also shown and depends on $\theta$ and $\varphi$ and on the goniometer arrangement (beam divergence, slit aperture etc.) The part of Fig. 1 drawn in perspective shows the dimensions of the irradiated volume, which, when $\varphi=0$, is

$$
V_{\mathrm{irr}}^{b}(0, \varphi)=s(\theta) t \xi_{m} \sin \theta
$$

The evolution of these variables is not the same for a bulk $(b)$ as for a film $(f)$ sample and evidently does not lead to the same irradiated volume. As we can see in

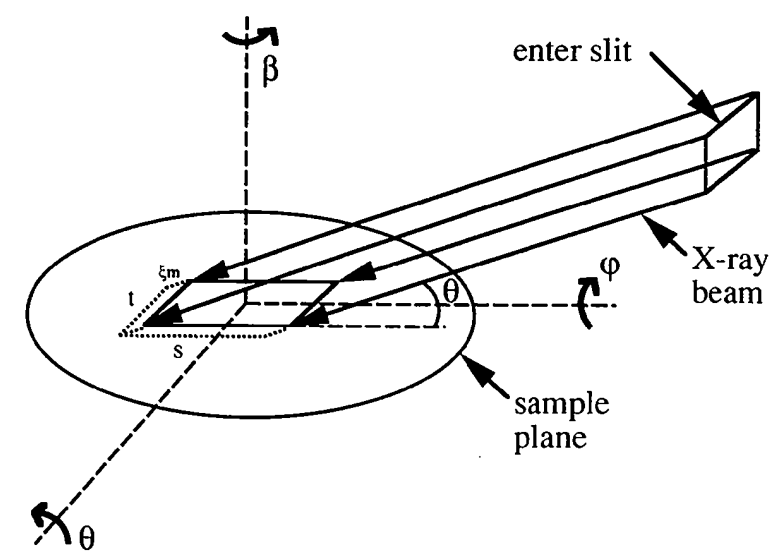

Fig. 1. Definition of the rotating axes in the Schulz reflection method. Rotation around the $\varphi$ and $\beta$ axes allows us to scan the direct space without the loss of the Bragg angle $\theta$, i.e. for the same $(h k l)$ diffracting planes. The irradiated volume is also defined, for an azimuthal angle $\varphi=0$.

(C) 1992 International Union of Crystallography 
Fig. 2, $V_{\text {irr }}^{b}$ is constant for a given $\theta$ and for all $\varphi$, since as $\varphi$ varies from 0 to $\varphi, t$ moves from $t$ to $t / \cos \varphi$ and $\xi_{m}$ (the penetration of the radiation) remains constant. So we can write

$$
V_{\mathrm{irr}}^{b}(\varphi, \theta)=s(\theta)(t / \cos \varphi) \xi_{m} \sin \theta \cos \varphi,
$$

where $s$ depends only on $\theta$ for a given apparatus configuration. Hence,

$$
V_{\mathrm{irr}}^{b}(\varphi, \theta)=V_{\mathrm{irr}}^{b}(0, \theta) .
$$

In the case of thin films, the thickness $e$ remains constant and the irradiated surface increases with $\varphi$, so the irradiated volume is

$$
V_{\mathrm{irr}}^{f}(\varphi, \theta)=s(\theta) e(t / \cos \varphi) .
$$

Hence,

$$
V_{\mathrm{irr}}^{f}(\varphi, \theta)=V_{\mathrm{irr}}^{f}(0, \theta) / \cos \varphi .
$$

It is interesting to note that if $e / \cos \varphi \geq \xi_{m}$ or $e / \sin \theta \geq \xi_{m}$ then we can treat the film sample as a bulk, i.e. make no special corrections. The first limit tells us that the evolution of the defocalization curve of a film becomes identical to that of a bulk for an azimuthal domain in $\varphi$ defined by

$$
\varphi \geq \operatorname{arcos}\left(e / \xi_{m}\right) .
$$

The second limit gives the domain of validity in $\theta$ inside of which (2), (5), (6) and (7) remain valid. In respect of this limit, we can consider a film if

$$
\theta \geq \arcsin \left(e / \xi_{m}\right)
$$

\section{Film corrections}

Let us proceed with this development taking into account the fact that the diffraction material is the same for both the film and bulk samples and that the substrate, or buffer, layer does not contribute to the diffracted intensity at a fixed $\theta$. For the same diffraction angle we can represent the ratio of the two

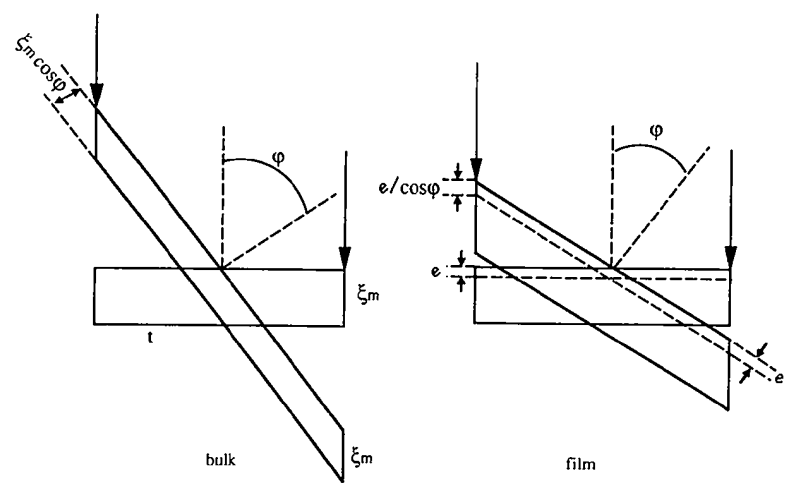

Fig. 2. Cross section of the irradiated volume for bulk and thin-film samples. volumes by that of the two diffracted intensities:

$$
I_{d}^{f}(0, \theta) / I_{d}^{b}(0, \theta)=V_{\mathrm{irr}}^{f}(0, \theta) / V_{\mathrm{irr}}^{b}(0, \theta)=e /\left(\xi_{m} \sin \theta\right) .
$$

From this and (1) and (2) we can also write

$$
I_{d}^{f}(\varphi, \theta)=\left[I_{d}^{f}(0, \theta) / \cos \varphi\right]\left[I_{d}^{b}(\varphi, \theta) / I_{d}^{b}(0, \theta)\right],
$$

where $I_{d}^{f}(\varphi, \theta)$ is the diffracted intensity from a randomly oriented film, $I_{d}^{f}(0, \theta)$ is this intensity at $\varphi=0, I_{d}^{b}(\varphi, \theta)$ is the diffracted intensity from a randomly oriented bulk and $I_{d}^{b}(0, \theta)$ is this intensity at $\varphi=0$. The combination of (5) and (6) gives the desired relation:

$$
I_{d}^{f}(\varphi, \theta)=e I_{d}^{b}(\varphi, \theta) /\left(\xi_{m} \sin \theta \cos \varphi\right) .
$$

This relation provides the defocalization correction curves for thin films in terms of those of the bulk, but it is only available under the aforementioned conditions and requires three sets of data: the correction curves for defocalization in the case of a bulk sample of the same material; the thickness of the film; and the radiation penetration depth in the material. These requirements are either generally known, or more or less easily obtained from experiment (bulk defocalization curves and $e$ ) or calculation $\left(\xi_{m}\right)$.

The irradiated volume being always smaller in a film and the decreasing evolution of the defocalization curve being compensated for by the $1 / \cos \varphi$ factor, the main results (see Fig. 3) are that the decrease in intensity is slower for a film (the defocalization is less effective) and that the correction needs to be less than for bulk samples.

\section{Substrate corrections}

The substrate must be considered separately since the X-ray beam is consistently absorbed across the

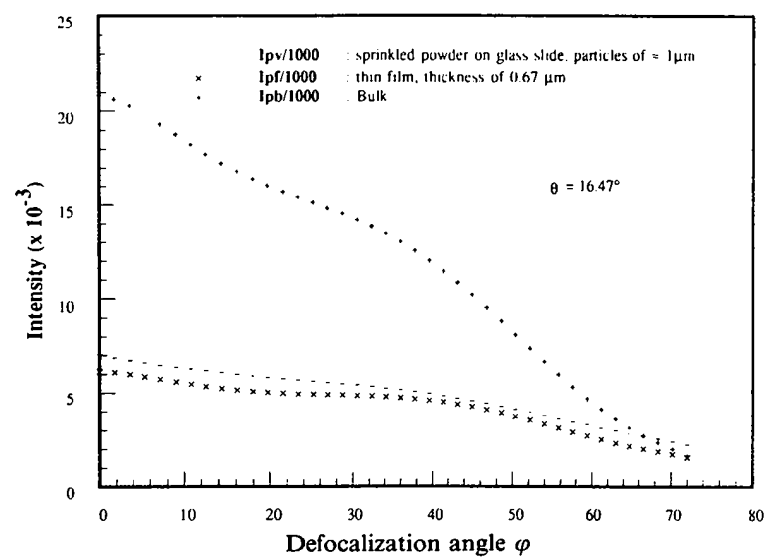

Fig. 3. Experimental defocalization curve for the 103 reflection of $\mathrm{YBa}_{2} \mathrm{Cu}_{3} \mathrm{O}_{7-\delta}$ bulk (top curve) and sprinkled powder (middle curve) and calculated curve for thin film (bottom curve). Intensities are corrected for background. 
film thickness before and after diffraction. In the following exposition, we assume that the substrate is a bulk (with no dependence of the irradiated volume on $\varphi$ ) and we make the same assumptions as before. In this way, the intensity diffracted by the substrate across the film, $I_{d}^{s}(\varphi, \theta)$, can be related to the intensity diffracted by a non-oriented bulk without film, $I_{d}^{b s}(\varphi, \theta)$, by

$$
I_{d}^{s}(\varphi, \theta)=I_{d}^{b s}(\varphi, \theta) \exp [-2 \mu x(\varphi, \theta)],
$$

where $x(\varphi, \theta)$ is the thickness of the film seen under $\varphi$ and $\theta$ and takes the value $e /(\cos \varphi \sin \theta)$ and $\mu$ is the linear absorption coefficient of the film. Thus, the defocalization curves for correcting substrates may be calculated from those of non-oriented bulk samples by the relation

$$
I_{d}^{s}(\varphi, \theta)=I_{d}^{b s}(\varphi, \theta) \exp [-2 \mu e /(\cos \varphi \sin \theta)] .
$$

For a given material and a Bragg angle $\theta, I^{s}(\varphi, \theta)$ decreases as $\varphi$ increases and the defocalization effect is enhanced compared with the buik. Furthermore, the allowed experimental zone for a substrate is reduced in both $\varphi$ and $\theta$ with the Schulz reflection method, in agreement with (3) and (4).

\section{Buffer-layer corrections}

The problem caused by the buffer layer is in fact a combination of the two preceding effects, the diffracted intensity modification being both reduced by absorption and enhanced by the increase in volume. Combining these two effects with the help of (7) and (8) gives the diffracted intensity correction for a buffer layer, $I_{d}^{\mathrm{bl}}(\varphi, \theta)$, in terms of that for a bulk, $I_{d}^{\mathrm{bb}}(\varphi, \theta)$ :

$$
\begin{aligned}
I_{d}^{\mathrm{bl}}(\varphi, \theta)= & {\left[e^{\prime} I_{d}^{\mathrm{bb}}(\varphi, \theta) / \xi_{m}^{\prime} \sin \theta \cos \varphi\right] } \\
& \times \exp (-2 \mu e / \sin \theta \cos \varphi),
\end{aligned}
$$

where $e^{\prime}$ and $\xi_{m}^{\prime}$ are the thickness of and X-ray penetration depth in the buffer layer, respectively.

With (3) and (4), two other limiting conditions allow the layers to be considered as either a bulk or a film:

$$
\varphi<\arccos \left(e^{\prime} / \xi_{m}^{\prime}\right) \text { and } \theta \geq \arcsin \left(e^{\prime} / \xi_{m}^{\prime}\right)
$$

\section{Corrections for a multilayer sample}

For a multilayer, each layer must be treated separately from the others. If $n$ is the number of layers, then for the $j$ th one considered for defocalization calculation we can modify (9) as follows:

$$
\begin{aligned}
I_{d_{j}}^{\mathrm{bl}}(\varphi, \theta)= & {\left[e_{j} I_{d_{j}}^{\mathrm{bb}}(\varphi, \theta) / \xi_{m_{j}} \sin \theta \cos \varphi\right] } \\
& \times \exp \left[\left(-2 \sum_{i=1}^{j-1} \mu_{i} e_{i}\right) / \sin \theta \cos \varphi\right],
\end{aligned}
$$

taking into account (3) and (4) for each layer. The result is that the available experimental domain in $\varphi$ decreases as the depth of the layer under consideration increases.

The substrate can be considered as the $j$ th and last bulk layer with respect to (8) which gives here:

$$
I_{d_{j}}^{s}(\varphi, \theta)=I_{d}^{\mathrm{bs}}(\varphi, \theta) \exp \left[\left(-2 \sum_{i=1}^{j-1} \mu_{i} e_{i}\right) / \sin \theta \cos \varphi\right]
$$

\section{Experimental procedure}

In this case, the method employed for the calculation of standardized intensities for a bulk sample is the usual one, as in previous work (Tenckhoff, 1970; Huijser-Gerits \& Rieck, 1974; Holland, 1964):

$$
\begin{aligned}
I_{\text {stand }}(\varphi, \beta, \theta)= & \left\{I_{\text {meas }}(\varphi, \beta, \theta)\right. \\
& \left.-I_{\mathrm{bkgr}}\left[I_{\mathrm{bkgr} / \mathrm{rand}}(\varphi, \theta) / I_{\mathrm{rand}}(0, \theta)\right]\right\} \\
& \times\left[I_{\mathrm{rand}}(\varphi, \theta)-I_{\mathrm{bkgr} / \text { rand }}(\varphi, \theta)\right]^{-1} \\
& \times\left[I_{\mathrm{rand}}(0, \theta)-I_{\mathrm{bkgr} / \text { rand }}(0, \theta)\right],
\end{aligned}
$$

where $I_{\text {stand }}(\varphi, \beta, \theta)$ is the calculated standardized intensity at spatial position $(\varphi, \beta), I_{\text {meas }}(\varphi, \beta, \theta)$ is the experimental intensity of the textured specimen, $I_{\mathrm{bkgr}}$ is the average of the background intensities of the textured specimen on both sides of the Bragg peak for the same integration time as $I_{\text {meas }}(\varphi, \beta, \theta), I_{\text {rand }}(\varphi, \theta)$ is the intensity of the random specimen (film or bulk) measured at $\theta, I_{\mathrm{bkgr} / \mathrm{rand}}(\varphi, \theta)$ is the background intensity of the latter specimen for the same integration time and $I_{\text {rand }}(0, \theta)-I_{\mathrm{bkgr} / \mathrm{rand}}(0, \theta)$ is the difference between the two previous intensities at $\varphi=0$.

In the case of a film on a substrate and buffer layer, $I_{\text {rand }}(\varphi, \theta)$ is determined by (7), (8) and $(9) . I_{\mathrm{bkgr} / \mathrm{rand}}(\varphi, \theta)$ has been estimated homothetically for a bulk evolution, since for all $\theta$ both substrate and film contributions are present in the background. This procedure seems to provide acceptable results, but more details are required of the background evolution, taking into account the perfectly oriented substrate.

\section{Experimental results}

Our theoretical results were investigated for a $\mathrm{YBa}_{2} \mathrm{Cu}_{3} \mathrm{O}_{7-\delta}$ thin film grown by laser ablation on a (100)-oriented $\mathrm{SrTiO}_{3}$ single crystal. The penetration depth in $\mathrm{YBa}_{2} \mathrm{Cu}_{3} \mathrm{O}_{7-\delta}$ is about $8 \mu \mathrm{m}$ and the film thickness is $0.67 \mu \mathrm{m}$.

First, we measured the 200 pole figure of $\mathrm{YBaCuO}$ which showed that all the crystallites have their $a$ axis perpendicular to the film surface (Fig. 4). So we focused our attention on the 103/110-type directions. The defocalization curves for film and bulk for these reflections are compared in Fig. 3. In this case the normalization factor of $(12), \quad\left[I_{\text {rand }}(0, \theta)-\right.$ 
$\left.I_{\mathrm{bkgr} / \mathrm{rand}}(0, \theta)\right] /\left[I_{\mathrm{rand}}(\varphi, \theta)-I_{\mathrm{bkgr} / \mathrm{rand}}(\varphi, \theta)\right]$, is approximately twice as large in a bulk as in a film at $\varphi=60^{\circ}$.

This behaviour is shown in Fig. 4. In Fig. 4(a), we present the 103 pole figure when intensity corrections for the bulk are used. We can see four poles well indexed as $103,1 \overline{1} 0,10 \overline{3}$ and 110 at $\sim 60^{\circ}$ from one another. Three further poles are also present, indicated as 1, 2 and 3 on the figure. The latter cannot be attributed to $\mathrm{YBa}_{2} \mathrm{Cu}_{3} \mathrm{O}_{7-\delta}$ or to the substrate, owing to their angular disposition, or to predominant parasitic phases noticed in a $\theta-2 \theta$ scan (not shown here). In fact, the explanation lies in a sporadic increase of these extra poles caused by bad corrections, as remarked on previously, and partly in

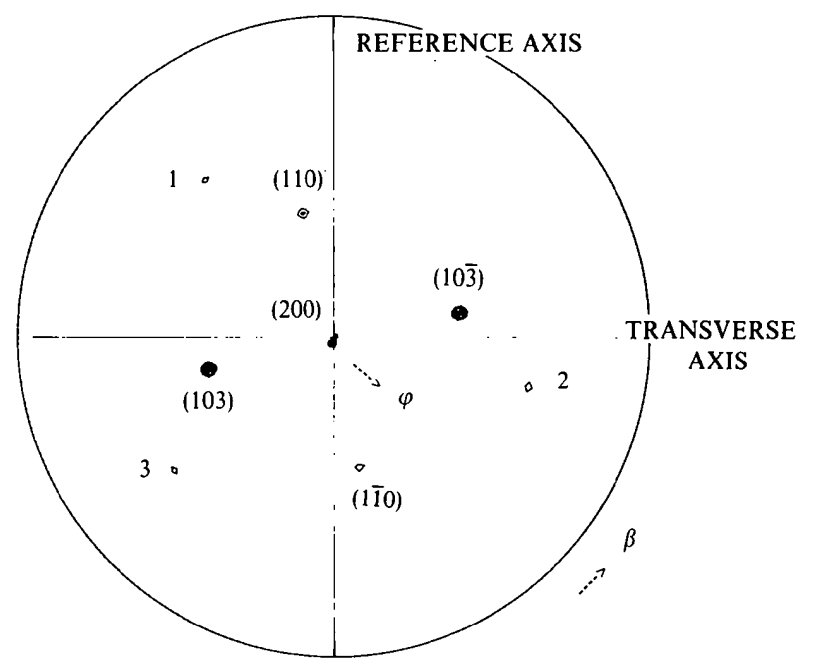

(a)

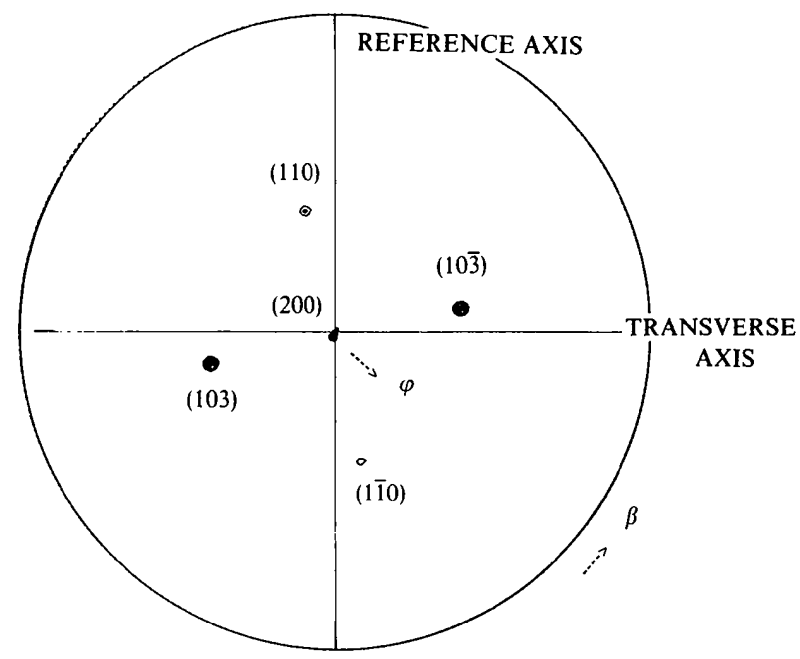

(b)

Fig. 4. Multipole figures of the 103- and 200-type reflections of $\mathrm{YBa}_{2} \mathrm{Cu}_{3} \mathrm{O}_{7-\delta}$ on a (100)-oriented $\mathrm{SrTiO}_{3}$ single crystal $(a)$ with a bulk defocalization correction and $(b)$ with the defocalization corrected for a film. a minor phase and/or an irregular background (irregular thickness of the film).

In Fig. 4(b), we have applied the calculated film defocalization curve. The same 103-type poles appear, with correctly modified pole intensities and without any parasitic poles. The excessive enhancement of the latter has disappeared, relegating them to below $15 \%$ of the maximum intensity.

\section{Accuracy evaluation for thin-film corrections}

In order to estimate the validity of our corrections for films, and since no randomly oriented ones could be fabricated, we have used as substitute a sprinkled powder sample on a glass slide. The small grains (about $1 \mu \mathrm{m}$ ) of $\mathrm{YBa}_{2} \mathrm{Cu}_{3} \mathrm{O}_{7-\delta}$ were randomly distributed on Vaseline, as densely as possible, with care taken to produce only one layer of grains.

Fig. 3 compares the experimental results obtained for such a sample with the calculated corrections for a bulk sample. The two are in good agreement as regards their $\varphi$ evolution with, however, a small difference in curvature for $\varphi$ 's in the middle of the range, which is probably attributable to the difference in sample compaction. We can also see an intensity shift which may be explained by a change in the diffracted volume owing to thickness (grain sizes) and, again, differences in the degree of compaction.

\section{Concluding remarks}

We have described a new method for correcting the experimental data of Schulz reflection texture analysis for the case of thin films in different configurations. The first results are encouraging and show the potential of larger quantitative use of this method. This work avoids the very arduous fabrication of non-oriented standards for each thickness of film and seems to be of more use in direct studies of layer epitaxy. The limit of such studies is related to the incident-beam penetration depth and layer thickness.

This work was partly supported by AlcatelAlsthom Research (Laboratoires de Marcoussis, France).

\section{References}

Bunge, H. J. (1982). Quantitative Texture Analysis, edited by H. J. Bunge, p. 85. Oberursel: Deutsche Gesellschaft für Metallkunde e.V.

Hermida, J. D. (1982). Mater. Sci. Eng. 56, 135-141.

Holland, J. R. (1964). Adv. X-ray Anal. 7, 86-93.

Huijser-Gerits, E. M. \& Rieck, G. D. (1974). J. Appl. Cryst. 7, 286-290.

Humbert, M. (1986). Experimental Techniques of Texture Analysis, edited by H. J. Bunge, p. 29. Oberursel, Deutsche Gesellschaft für Metallkunde e.V.

Ortiz, M. \& Hermida, J. D. (1981). Texture, 4, 159-169.

Schulz, L. G. (1949). J. Appl. Phys. 20, 1030-1037.

Tenckhoff, E. (1970). J. Appl. Phys. 41, 3944-3948. 